\title{
Análisis de métodos de diseño de pavimentos de adoquines de hormigón
}

\author{
Assessment of pavement concrete blocks design methods
}

Rodrigo Bahamondes (Autor Principal)

rbahamondes@udec.cl

Universidad de Concepción, Facultad de Ingeniería

Depto. de Ing. Civil, Chile

Fono +56-41-2203604, Fax +56-41-2207089

Edmundo Larenas 219, Concepción, Chile

Tomás Echaveguren (Autor Oficial de Contacto)

Universidad de Concepción, Chile

techaveg@udec.cl

\section{Resumen}

Los pavimentos de adoquines de hormigón (PAH) se utilizan como una solución alternativa a los pavimentos tradicionales para calzadas vehiculares de baja velocidad. En este trabajo se analizaron los métodos de diseño de PAH propuestos en Australia, Japón, Estados Unidos, Reino Unido e India. Se utilizó un rango de CBR de la subrasante entre $4 \%$ y $20 \%$ y niveles de tránsito entre 45.000 y 4.500 .000 ejes equivalentes acumulados. Luego se estimaron como parámetros de desempeño tensiones, deformaciones y deflexiones de la subrasante, las que se contrastaron con valores admisibles. Los resultados mostraron que para $C B R>10 \%$ y niveles de tránsito bajos, todos los diseños convergieron a un diseño mínimo que cumplió además con los 3 parámetros de desempeño utilizados. Se determinó que para niveles mayores de tránsito y $\mathrm{CBR}<10 \%$, los métodos del Reino Unido y Japón proporcionan los mejores desempeños.

Keywords: pavement design; concrete blocks; stress, strains, deflections

Introducción

Los pavimentos representan una parte importante de los activos viales. Deben ser capaces de soportar solicitaciones de tránsito, ambientales y proveer una estructura durable que permita la circulación de los vehículos con comodidad y seguridad. En el diseño de pavimentos tradicionalmente se han usado tecnologías de diseño basadas en pavimentos rígidos y flexibles, masificados en todo el mundo a partir del ensayo AASHO realizado en 1956 en Estados Unidos (AASHTO, 1993). Los pavimentos de adoquines han estado presentes en la ingeniería de pavimentos desde la época del Imperio romano. Pero no es sino hasta la década de 1970 en donde comienza a masificarse el uso de adoquines de hormigón estacionamientos, calzadas vehiculares, peatonales y espacios públicos. Esto llevó a que diversos países iniciaran la investigación en busca de sus propios métodos de diseño de pavimentos de adoquines. Entre ellos Holanda, Australia y el Reino Unido. En la actualidad existen alrededor de 12 métodos de diseño (Morrish, 1980; Miura et al., 1984; Rollings, 1984; Houben et al., 1986; Clifford, 1987; Livneh et al., 1988; Eisenmann \& Leykauf, 1988; Rada et al., 1990; Judycki et al.,

\author{
Sergio Vargas-Tejeda \\ Universidad del Bío Bío, Chile \\ svargas@ubiobio.cl
}

\section{Abstract}

The pavements of concrete pavers (PAH) are used as an alternative to traditional paving of low speed driveways. This paper analyze the PAH design methods proposed in Australia, Japan, United States, United Kingdom and India. A range of CBR subgrade between $4 \%$ and $20 \%$ and traffic levels between 45,000 and 4,500,000 cumulative equivalent axles were used. The performance parameters for design were the stresses, strains and deflections of the subgrade, which were compared with allowable values. Results showed that for CBR> $10 \%$ and low traffic levels, all designs converged to a minimum design and also accomplished with the 3 performance parameters used in this research. It was determined that higher levels of traffic and $C B R<10 \%$, the methods of the United Kingdom and Japan provides the best performance.

1996; Silfwerbrand \& Wäppling, 2000; BSI, 2001a; BSI, 2001b; Ryntathiang et al., 2006).

Los países que no cuentan con un método de diseño propio se ven en la necesidad de adoptar algunos de estos métodos. Esto no necesariamente garantiza su validez al aplicarlo bajo condiciones distintas a aquellas donde el método fue desarrollado y calibrado, ni tampoco asegura un desempeño adecuado del pavimento. Por tanto, una de las tareas esenciales previas a la adopción de un método de diseño es explorar los resultados de diseño frente a condiciones locales $y$, a la vez, evaluar de una forma sistemática su desempeño potencial. Esto constituye una primera etapa en el camino hacia una calibración local de un método de diseño.

Este artículo tiene por objetivo explorar los métodos de diseño de pavimentos de adoquines más relevantes del estado del arte, para así evaluar el desempeño potencial ante diversos escenarios de diseño y emitir así recomendaciones orientadas a la futura calibración de un método de diseño de pavimentos de adoquines. Para ello, en primer lugar se describen los componentes y comportamiento estructural de los pavimentos de adoquines de hormigón. Luego se describen los métodos de 
diseño de Australia, Japón, Estados Unidos, Reino Unido e India con las consideraciones y supuestos de cada uno para modelar y diseñar. Posteriormente se utilizó cada método de diseño para obtener espesores de las capas granulares, para un total de 25 escenarios de diseño dependientes del CBR de la subrasante y de la solicitación de tránsito. Finalmente se realiza un análisis del desempeño de los diseños obtenidos con los diversos métodos mediante un análisis mecanicista.

\section{Los pavimentos de adoquines de hormigón}

Los pavimentos de adoquines se componen de distintas capas al igual que los pavimentos rígidos y flexibles. La principal diferencia con estos últimos radica en la composición de la carpeta de rodadura, que está conformada por adoquines intertrabados, que brindan al pavimento un comportamiento estructural semiflexible. De este modo, los pavimentos de adoquines están constituidos por una capa de adoquines, arena de juntas, una cama de arena, base y subbase (Figura 1). Poseen también un borde de confinamiento que contribuye al desarrollo del mecanismo de trabazón mecánica.

Figura 1. Elementos estructurales de un pavimento de adoquines (ASCE, 2010).

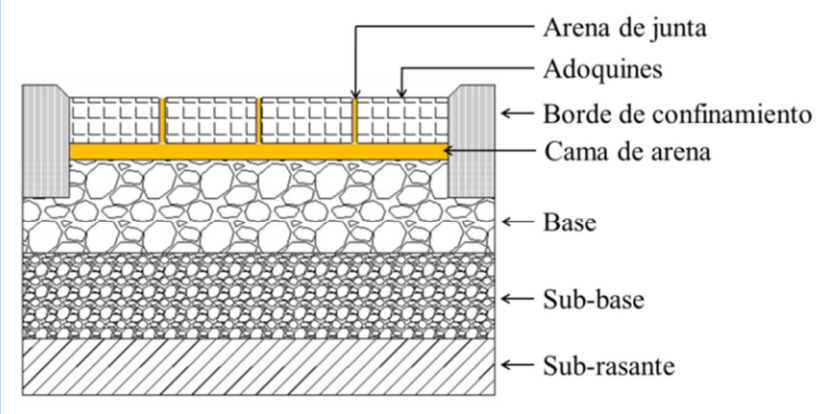

El adoquín constituye la superficie de rodadura. Su espesor varía entre 50 y $100 \mathrm{~mm}$ dependiendo de las solicitaciones de carga (Morrish, 1980; BSI, 2001a). Su resistencia a la compresión varía entre 25 y $45 \mathrm{MPa}$ (Morrish, 1980; ASCE, 2010). La arena de las juntas se utiliza para rellenar los intersticios entre adoquines para favorecer la trabazón mecánica entre las caras laterales. El espesor de los intersticios varía entre 2 y $5 \mathrm{~mm}$ (Panda \& Gosh, 2002). La cama de arena es una superficie de nivelación para la instalación de los adoquines, cuyo espesor varía entre 20 y $30 \mathrm{~mm}$ compactados (Rada et al., 1990; Shackel et al., 1993).

Durante la compactación, parte de la arena asciende entre las juntas, con lo cual colabora con la trabazón mecánica entre los adoquines. Las capas de base y subbase disipan las tensiones producidas por las cargas de tránsito transmitidas desde la carpeta de rodadura, de tal forma que en la subrasante no se superen las tensiones admisibles. La especificación chilena para pavimentos carreteros recomienda un CBR mínimo de $80 \%$ para bases granulares y un CBR mínimo de $40 \%$ para subbases granulares (MOP, 2012). Para pavimentos urbanos, la especificación chilena recomienda un CBR mínimo de $80 \%$ para bases granulares y un CBR mínimo de $35 \%$ para subbases granulares (MINVU, 2008).

\section{Comportamiento estructural de los pavimentos de adoquines}

Los adoquines, arena de juntas y cama de arena generan un mecanismo de trabazón mecánica entre los adoquines mediante el cual son capaces de disipar tensiones, transmitiendo carga entre adoquines adyacentes. Shackel \& Lim (2003) describen el mecanismo de trabazón mecánica como el resultado del efecto combinado de cuña y rotación que se produce entre adoquines al ser cargados. En el efecto de cuña el adoquín cargado empuja longitudinalmente los adoquines vecinos. El efecto de rotación, en tanto, hace girar los adoquines vecinos en torno a alguno o varios de sus ejes. En adoquines rectangulares se produce el efecto de cuña o el efecto combinado de cuña y rotación en el caso de adoquines de caras dentadas. A lo anterior se agrega el aporte de la fricción entre las caras laterales de los adoquines, inducido por la arena en las juntas.

Otro aspecto particular de los pavimentos de adoquines es que la trabazón mecánica aumenta con la aplicación de cargas verticales hasta llegar a un nivel de equilibrio. Este se alcanza aproximadamente después de 10.000 repeticiones de carga de un eje estándar (ejes equivalentes acumulados, EEA) (Houben et al., 1986; Rada et al., 1990). Este comportamiento tiene asociado un aumento en la rigidez de la carpeta de rodadura, con lo cual aumenta su capacidad de disipar tensiones y consecuentemente disminuyen las tensiones que se transmiten a las capas inferiores del pavimento. Houben et al. (1986) verificaron este aumento de la rigidez a través de mediciones en tramos de prueba y lo asociaron al comportamiento de las deformaciones permanentes del pavimento. Propusieron un modelo de progresión de las deformaciones permanentes en función de EEA de $80 \mathrm{kN}$.

El modelo de Houben et al. (1986), plantea que las deformaciones elásticas mantienen un nivel constante mientras se desarrolla la trabazón mecánica, hasta que alcanzan su nivel de equilibrio, lo cual ocurre cuando los ejes equivalentes acumulados alcanzas un valor crítico Luego las deformaciones elásticas disminuyen de manera progresiva. Asimismo, las deformaciones permanentes aumentan a una tasa de acumulación decreciente. Houben et al. (1986) utilizaron su modelo de progresión de las deformaciones permanentes para proponer un método de diseño para las condiciones de suelo y materiales de Holanda, usando como criterio de diseño el ahuellamiento en el pavimento. Otros métodos también consideran la rigidización de la carpeta de rodadura. Rada et al. (1990), proponen el aumento en el valor del coeficiente estructural hasta llegar a 10.000 EEA, a partir del cual dicho coeficiente permanece constante. Silfwerbrand y Wäppling (2000) propusieron una progresión del módulo de elasticidad 
de la capa desde $1.500 \mathrm{MPa}$ hasta $6.000 \mathrm{MPa}$, valor que se alcanza con 10.000 EEA.

\section{Métodos de diseño de pavimentos de adoquines de hormigón}

Los métodos de diseño se clasifican en: los basados en la capacidad de soporte del suelo, métodos empíricos, de secciones normalizadas y mecanicistas. Ejemplos de métodos de diseño según esta clasificación son los propuestos en Australia (Morrish, 1980), Japón (Miura et al., 1984), Estados Unidos (Rada et al., 1990), Reino Unido (BSI, 2001a y b) e India (Ryntathiang et al., 2006). Los métodos basados en la capacidad de soporte del suelo fueron los primeros en desarrollarse. El cálculo del espesor de la base $\left(H_{b}\right)$ para un cierto nivel de tránsito, espesor de adoquín y cama de arena, se realiza en función del CBR de la subrasante usando un modelo que relaciona linealmente el $\mathrm{CBR}$ y $\mathrm{H}_{\mathrm{b}}$. Dentro de este enfoque se encuentra el método propuesto en Australia (Morrish, 1980) adoptado en Chile (Barthou, 1991) y también el propuesto en Israel (Livneh et al., 1988).

Dentro de los métodos empíricos se encuentra el método de Rada et al. (1990), que utiliza la ecuación AASHTO de diseño de pavimentos flexibles. Este método se utiliza actualmente en Estados Unidos (ASCE, 2010). En Japón, Miura et al. (1984) propusieron un método similar que asume un comportamiento asimilable al de pavimentos flexibles. Otro de los enfoques de diseño es el mecanicista clásico, en el que se calcula el estado de tensiones y el ciclo de fatiga del material en un sistema multicapa, para así determinar los espesores de las capas de base y/o subbase que cumplen con el criterio de fatiga. Dentro de estos métodos se encuentran los de Suecia (Silfwerbrand \& Wäppling, 2000) e India (Ryntathiang et al., 2006). En los métodos de secciones normalizadas, el diseño del espesor de las capas del pavimento se realiza en base a catálogos de secciones tipo obtenidas previamente aplicando en forma repetitiva métodos de diseño mecanicista, como es el caso de Polonia (Judycki et al., 1996), Sudáfrica (Clifford, 1987), Alemania (Eisenmann \& Leykauf, 1988) y Reino Unido (BSI, 2001a y b).

\section{Método de diseño de Australia}

Este método utiliza curvas de diseño calibradas para condiciones locales. Considera cinco niveles de tránsito para calzada vehicular $(A, B, C, D$ y E) y dos niveles para tránsito industrial $(X$ e $Y$ ). Cada nivel de tránsito tiene asociada una curva de diseño, como muestra la Figura 2. Para determinar el espesor de la base granular, se ingresa al gráfico de la Figura 2 con el valor de CBR de la subrasante y se intersecta la curva correspondiente al nivel de tránsito de diseño seleccionado. El resultado es el espesor mínimo de la base granular. El método no utiliza indicadores de desempeño, por lo que se asume que el criterio de diseño es la falla de la subrasante. El método es aplicable hasta una solicitación de 4.500.000 EEA.

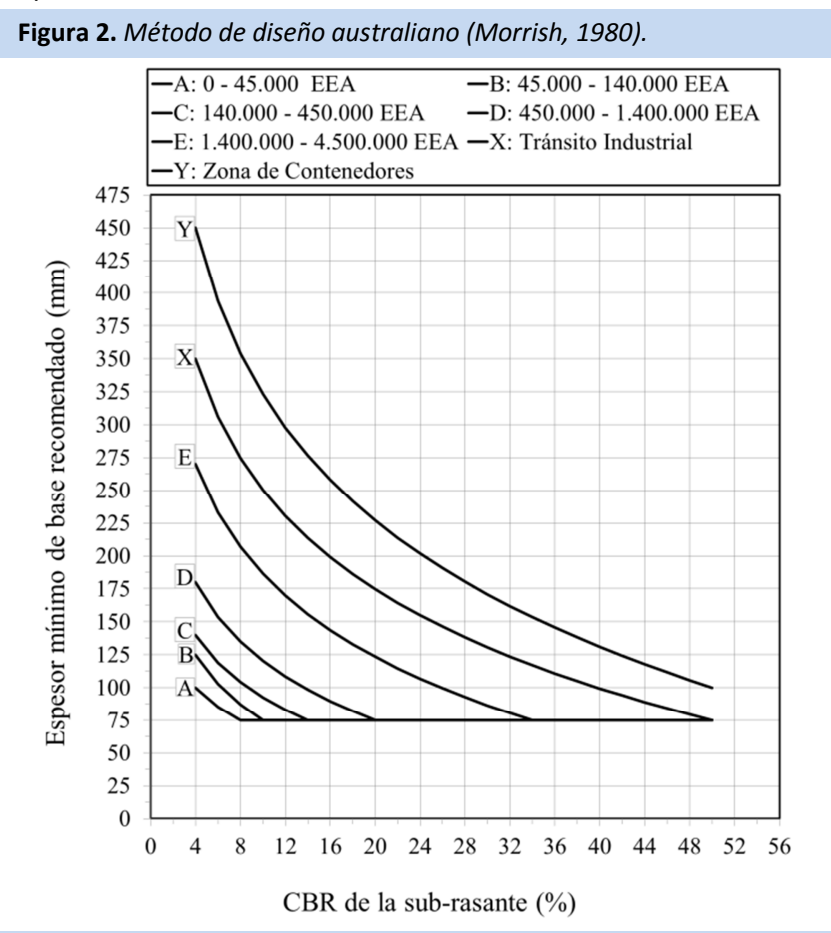

\section{Método de diseño de Japón}

Este método adaptó el método de diseño de pavimentos flexibles de Japón para diseñar pavimentos de adoquines a través del coeficiente de resistencia relativa de la capa de adoquines. Por tanto, asume que el comportamiento de los pavimentos de adoquines es similar al de los pavimentos flexibles. El método establece que tanto los materiales como el espesor de cada capa deben satisfacer las ecuaciones 1, en las cuales el espesor total del pavimento $(H)$ está en función del CBR de la subrasante y del número de pasadas equivalentes acumuladas de un eje estándar de $98 \mathrm{kN}\left(\mathrm{N}_{98}\right)$. El espesor de mezcla asfáltica equivalente expresado como número estructural (NE) está en función del CBR y de $\mathrm{N}_{98}$, y se calcula como la suma del producto de los espesores de capa $h_{i}$ por los coeficiente de resistencia relativa del material $\left(a_{i}\right)$. EI coeficiente de resistencia relativa de la capa de adoquines $\left(a_{1}\right)$ se obtiene en función del módulo de elasticidad del material de la capa "i” ( $E_{i}$, en kg/cm²) (Miura et al., 1984).

$$
\begin{aligned}
& H=28 \frac{N_{98}^{0,1}}{C^{0} R^{0,6}} ; N E=3,84 \frac{N_{98}^{0,16}}{C^{0,0,3}} \\
& N E=a_{1} h_{1}+a_{2} h_{2}+\ldots .+a_{n} h_{n}=\sum a_{i} h_{i} \\
& a_{1}=0,00525\left(\frac{E_{i}^{0,46}}{0,44}\right)
\end{aligned}
$$




\section{Método de diseño de Estados Unidos}

El método fue propuesto originalmente por Rada et al. (1990). Al igual que el método japonés, este método asume que un pavimento de adoquines tiene un comportamiento similar al de un pavimento flexible. Por tanto, asume que la pérdida de serviciabilidad del pavimento de adoquín se puede asimilar a la de un pavimento flexible. De este modo, utiliza la ecuación AASHTO 1986 (Ecuación 2) para pavimentos flexibles, caracterizando a los adoquines mediante el coeficiente estructural de Takeshita (Miura et al., 1984).

$$
\begin{aligned}
& N_{80}=(N E+24,5)^{9,36} 10^{-\left(16,4+Z_{R} s_{0}\right)} M_{R}^{2,32}\left(\frac{p_{i}-p_{f}}{p_{i}-1,5}\right)^{\frac{1}{\beta}} \\
& \beta=0,4+\left(\frac{97,81}{N E+25,4}\right)^{5,19}
\end{aligned}
$$

$$
E_{i+1}=0,2 h^{0,45} E_{i}
$$$$
M_{R}=\left\{\begin{array}{ccc}
10(C B R) & ; & C B R \leq 5 \% \\
17,6(C B R)^{0,65} & ; & C B R>5 \%
\end{array}\right.
$$

$$
\mathrm{N}_{80}=\frac{3,06 \times 10^{-7}}{\varepsilon_{z}^{4,5337}}
$$

En la ecuación $2 \mathrm{~N}_{80}$ es función de $\mathrm{NE}$, del coeficiente estadístico de la distribución normal $\left(Z_{R}\right)$, de la desviación estándar del error combinado de todas las variables $\left(\mathrm{S}_{0}\right)$, del módulo resiliente de la subrasante $\left(\mathrm{M}_{R}\right.$, en $\left.\mathrm{MPa}\right)$, del índice de serviciabilidad inicial y final del pavimento $\left(p_{i}\right.$ y $p_{f}$ respectivamente). El método considera el aporte estructural de la capa combinada de adoquines y cama de arena a través del coeficiente de capa $\left(a_{1}\right)$, el cual depende del módulo de elasticidad, del nivel de tránsito, y del tiempo de asentamiento. Este coeficiente aumenta linealmente desde 0,26 hasta 0,44, cuando la solicitación alcanza las 10.000 EEA. A partir de ese valor de solicitaciones el coeficiente de capa permanece constante y vale 0,44 .

\section{Método de diseño de India}

Este método considera el pavimento de adoquines como un sistema multicapa elástico. Cada capa de espesor $\left(h_{\mathrm{i}}\right)$ se caracteriza a través del módulo de elasticidad $\left(\mathrm{E}_{\mathrm{i}}\right)$ y del módulo de Poisson. Ryntathiang et al. (2006), mediante ensayos de placa de carga, estimaron el módulo de elasticidad de la capa de adoquines en 1.500 MPa. El módulo de elasticidad de las capas de base y sub-base granulares la estiman aplicando las Ec. 3, en las cuales primero se relaciona el módulo de elasticidad de la capa superior e inferior $E_{i+1}$ y $E_{i}$ (en MPa) con el espesor de la capa inferior ( $h$, en $\mathrm{mm}$ ) (Read \& Whiteoak, 2003). Luego el módulo resiliente de la sub-rasante lo estiman en función de las características mecánicas de todas las capas, para posteriormente calcular el estado de tensiones de la subrasante. Luego determina el número de repeticiones de carga de $80 \mathrm{kN}\left(\mathrm{N}_{80}\right)$ que admite el pavimento tal que la deformación de la subrasante $\left(\varepsilon_{z}\right)$ no exceda los $20 \mathrm{~mm}$.

\section{Método de diseño del Reino Unido}

Este método posee tabulados espesores de base y subbase en función de las solicitaciones de tránsito y CBR (Tablas 1 y 2 respectivamente). Estos espesores los obtuvieron en base a un análisis mecanicista en cual estandarizaron los materiales de la base de modo tal que reducir hasta un valor admisible preestablecido las deformaciones permanentes. El método considera bases granulares mejoradas con cemento pero con una resistencia a la compresión similar a la de hormigones ligeros que en general son superiores a los $6 \mathrm{MPa}$ ). Para obtener el espesor equivalente a bases de otro material, se

\begin{tabular}{|c|c|c|c|c|}
\hline \multirow{2}{*}{$\begin{array}{l}\text { Espesor de } \\
\text { capa }(\mathrm{mm})\end{array}$} & \multicolumn{4}{|c|}{$\begin{array}{l}\text { Espesor }(\mathrm{mm}) \text { de la base granular según tránsito } \\
\left(\operatorname{EEAx} 10^{6}\right)\end{array}$} \\
\hline & $0,5-1,5$ & $1,5-4,0$ & $4,0-8,0$ & $8,0-12,0$ \\
\hline Base granular & 390 & $390-480$ & 540 & $690-750$ \\
\hline $\begin{array}{l}\text { Base tratada } \\
\text { con cemento }\end{array}$ & 130 & $130-160$ & 180 & $230-250$ \\
\hline
\end{tabular}
multiplica por un factor de equivalencia que es igual a 3 para bases granulares con CBR=80 \% (Knapton, 2008).

Tabla 1. Espesor de la base (adaptado de BSI, 2001b)

Tabla 2. Espesor de la sub-base (adaptado de BSI, 2001a)

\begin{tabular}{l|l|l|l|l|l|l}
\hline \multirow{2}{*}{$\begin{array}{l}\text { Nivel de } \\
\text { tránsito } \\
\text { (EE/día) }\end{array}$} & \multicolumn{6}{l}{$\begin{array}{l}\text { Espesor }(\mathrm{mm}) \text { de la subbase granular según el CBR (\%) de } \\
\text { la subrasante }\end{array}$} \\
\cline { 2 - 7 } & 2 & 3 & 4 & $5-10$ & $10-15$ & $15-30$ \\
\hline$<60$ & 250 & 190 & 160 & 150 & 150 & 150 \\
\hline$<200$ & 310 & 240 & 210 & 180 & 150 & 150 \\
\hline$<500$ & 350 & 270 & 230 & 200 & 160 & 150 \\
\hline$<1.000$ & 400 & 310 & 270 & 225 & 180 & 150 \\
\hline$>1.000$ & 450 & 350 & 310 & 270 & 240 & 225 \\
\hline
\end{tabular}

\section{Diseño de estructuras de pavimento}

Se diseñaron distintas estructuras de pavimento usando los métodos de diseño antes descritos y compararlos. Para esto se caracterizaron los materiales de las capas del pavimento y se definieron los escenarios de diseño compatibles para todos los métodos, de acuerdo a los espacios de inferencia de las variables de entrada. La Tabla 3 resume las características de los materiales, niveles de solicitación de tránsito y criterios de diseño de los métodos estudiados. Estos métodos poseen espacios de inferencia comunes en cuanto a niveles de tránsito, capacidad de soporte del suelo, materiales y presencia de capas de base y la subbase. Por lo tanto, los diseños bajo dichas condiciones son comparables entre sí. 
Tabla 3. Caracterización de los métodos de diseño estudiados (Fuente: Elaboración Propia, 2012).

\begin{tabular}{|c|c|c|c|c|c|}
\hline \multirow{2}{*}{ Método } & \multicolumn{3}{|l|}{ Estructuración } & \multirow{2}{*}{ Tránsito (EEA) $\times 10^{6}$} & \multirow{2}{*}{ Criterio de diseño } \\
\hline & Espesor del adoquín (mm) & Base & Subbase & & \\
\hline Australia & $60,75,80$ y 100 & Granular & Sin subbase & $<4,5$ & Sin información \\
\hline Japón & $60,80,100$ & $\begin{array}{l}\text { Granular } \\
\text { Tratada con cemento } \\
\text { Tratada con asfalto }\end{array}$ & $\begin{array}{l}\text { Granular } \\
\text { Tratada con cemento }\end{array}$ & Sin Información & $\begin{array}{l}\text { Ahuellamiento } \\
<3,5 \mathrm{~cm}\end{array}$ \\
\hline $\begin{array}{l}\text { Estados } \\
\text { Unidos }\end{array}$ & 80 y 100 & $\begin{array}{l}\text { Granular } \\
\text { Tratada con cemento } \\
\text { Tratada con asfalto }\end{array}$ & $\begin{array}{l}\text { Granular } \\
\text { Tratada con cemento } \\
\text { Tratada con asfalto }\end{array}$ & $\begin{array}{l}\text { Máxima } \\
\text { solicitación según } \\
\text { AASHTO } 1986\end{array}$ & $\begin{array}{l}\text { Serviciabilidad } \\
\text { final de } 2,5\end{array}$ \\
\hline Reino Unido & $50,60,65$ y 80 & $\begin{array}{l}\text { Granular } \\
\text { Tratada con cemento } \\
\text { Tratada con asfalto }\end{array}$ & $\begin{array}{l}\text { Granular } \\
\text { Tratada con cemento }\end{array}$ & $0,5-12$ & $\begin{array}{l}\text { Deformación de la sub- } \\
\text { rasante }\end{array}$ \\
\hline India & 80 & Granular & Granular & 2,0 & $\begin{array}{l}\text { Deformación admisible } \\
\text { de la sub-rasante }\end{array}$ \\
\hline
\end{tabular}

\section{Estructuración y materiales del pavimento}

Las características de cada material se definieron de manera que sean compatibles para todos los métodos utilizados. Se adoptó un espesor de adoquín de $80 \mathrm{~mm}$, con una resistencia a la compresión de $35 \mathrm{MPa}$. A la capa de base granular se le asignó un CBR de 80\%. Se fijó un espesor mínimo de base de 75 mm de acuerdo a Morrish (1980) y Livneh et al. (1988). Se consideró únicamente un diseño con base, puesto que algunos métodos prescinden de la subbase granular. La subrasante se caracterizó usando un CBR entre $4 \%$ y $20 \%$. Valores inferiores al $4 \%$ corresponden a suelos orgánicos $(\mathrm{OH})$, arcillas de alta plasticidad $(\mathrm{CH})$ o limos de alta compresibilidad $(\mathrm{MH})$ según el sistema unificado de clasificación de suelos USCS. Por otra parte, para valores de CBR superiores al $20 \%$, la capacidad de soporte es suficientemente elevada como para que los diseños converjan a espesores mínimos.

\section{Cálculo de espesores de capas granulares}

Para realizar los diseños se construyó la matriz de diseño de la Tabla 4, según nivel de tránsito y $C B R$ de la subrasante, obteniéndose 25 escenarios de diseño. Para cada uno de ellos se obtuvo el espesor de la capa granular. En el caso del método de Australia el espesor de la base granular se obtuvo directamente de la Figura 2. En el caso del método de Japón, se utilizaron la ecuación 1 y un coeficiente de resistencia relativa de 0,35 (Miura et al., 1984). Para el método de Estados Unidos se utilizó un coeficiente estructural de la base de 0,14 (Rada et al., 1990). En ambos casos se consideró una capa granular.

En el caso del método del Reino Unido se calcularon los espesores de base y de subbase usando las Tablas 1 y 2 . Posteriormente se calculó el módulo de elasticidad de cada capa granular usando la ecuación 3. Con estos resultados y mediante la Ec. 4 la subbase granular se llevó a un espesor de base equivalente. El método de diseño británico calcula el espesor de la base usando una base estandarizada con contenido de cemento. Knapton (2008) recomienda amplificar por 3 dicho espesor para llevarlo a un espesor equivalente a una capa granular. Luego mediante la ecuación 4 se calculó el espesor equivalente de base granular en función del espesor de la sub-base. En dicha ecuación, $h_{2}$ es el espesor equivalente de la base granular respecto a la capa $h_{1}$ de la subbase, $E_{1}$ y $E_{2}$, son los módulos de elasticidad de la base y la subbase y $\mu_{1}, \mu_{2}$ corresponden al módulo de Poisson de las mismas capas.

Tabla 4. Escenarios de diseño según nivel de tránsito y CBR (Fuente: Elaboración Propia, 2012).

\begin{tabular}{l|l|l|l|l|l}
\hline \multirow{2}{*}{$\begin{array}{l}\text { CBR } \\
(\%)\end{array}$} & \multicolumn{5}{|l}{ Niveles de Tránsito $\left(\mathrm{EEA} / 10^{3}\right)$} \\
\cline { 2 - 6 } & 45 & 140 & 450 & 1.400 & 4.500 \\
\hline 4 & C4 N1 & C4 N2 & C4 N3 & C4 N4 & C4 N5 \\
\hline 6 & C6 N1 & C6 N2 & C6 N3 & C6 N4 & C6 N5 \\
\hline 8 & C8 N1 & C8 N2 & C8 N3 & C8 N4 & C8 N5 \\
\hline 10 & C10 N1 & C10 N2 & C10 N3 & C10 N4 & C10 N5 \\
\hline 20 & C20 N1 & C20 N2 & C20 N3 & C20 N4 & C20 N5 \\
\hline
\end{tabular}

$$
h_{2}=h_{1} \sqrt[3]{\frac{E_{1}}{E_{2}} \frac{1-\mu_{2}^{2}}{1-\mu_{1}^{1}}}
$$

Para el caso del método de diseño de India, el diseño mecanicista se realizó usando las ecuaciones 3 . Se consideró un espesor de base inicial de $75 \mathrm{~mm}$ para luego mediante iteraciones estimar la deformación vertical de la sub-rasante, y verificar el criterio de diseño usando la ecuación de cálculo de $\mathrm{N}_{80}$. De no cumplirse, se aumentó progresivamente el espesor de la base granular en $10 \mathrm{~mm}$ hasta cumplir con el criterio de diseño. En la Figura 3a (tránsito "bajo") se identifican los siguientes comportamientos: para valores de CBR inferiores a $10 \%$, exceptuando el método británico, los espesores divergen conforme el CBR disminuye hasta un valor máximo de $105 \mathrm{~mm}$ para un CBR del $4 \%$. Lo contrario ocurre para CBR mayores al $10 \%$, donde los espesores convergen hacia el espesor mínimo de $75 \mathrm{~mm}$. Por tanto, cuando la capacidad de soporte de la subrasante es elevada, diseñar con uno u otro método es indiferente. Por su parte, el método británico estima un espesor constante de $240 \mathrm{~mm}$ a partir del CBR del $6 \%$, lo cual se explica por el hecho que la normativa BSI recomienda el uso de bases y subbases lo cual incrementa los espesores. 
En la Figura 3b (tránsito "medio") se visualizan comportamientos similares a los observados en la Figura $3 a$. Para CBR inferiores al 10\% exceptuando el método británico, los espesores divergen a medida que el CBR es menor, hasta un valor máximo de $150 \mathrm{~mm}$ para un CBR del $4 \%$. En tanto que para CBR mayores al $10 \%$, la convergencia de los espesores es mayor a medida que el CBR aumenta, hasta alcanzar la convergencia total para un CBR del $20 \%$ con el diseño mínimo de $75 \mathrm{~mm}$. Para el escenario de tránsito "alto" (Figura 3c), los diseños se comportan de manera distinta al resto de niveles de tránsito analizados.

En efecto, los métodos de Australia e India convergen a los mismos resultados, obteniéndose en ambos casos espesores sistemáticamente inferiores en aproximadamente $90 \mathrm{~mm}$ y 220 $\mathrm{mm}$ respecto de los métodos de Estados Unidos y Japón respectivamente, para un CBR de $4 \%$. Los espesores obtenidos mediante el método británico se encuentran sistemáticamente por sobre el resto de los métodos. Esto se debe a que el factor de amplificación para bases granulares utilizado en el método del Reino Unido aumenta notoriamente el espesor de la base granular respecto de los otros métodos de diseño, para todo el rango de calidades de subrasante y niveles de tránsito.

\section{Análisis del estado de tensiones}

Con los diseños obtenidos en la sección anterior, se evaluaron las tensiones, deformaciones y deflexiones de la subrasante debido a la aplicación de la carga de rueda de un eje estándar. Esto con el objetivo de contar con indicadores cuantitativos del desempeño de los diseños realizados con cada método y poder así contrastarlos.

\section{Procedimiento de análisis}

Los pavimentos diseñados se modelaron como sistemas elásticos multicapas, mediante el software BISAR V 3.0 para el diseño mecanicista de pavimentos flexibles. Los supuestos del análisis fueron los siguientes: (a) cada sistema consta de capas horizontales de espesor uniforme, (b) las capas se extienden infinitamente en direcciones horizontales, (c) el material de cada capa es homogéneo e isotrópico, (d) los materiales son elásticos y tienen una relación lineal entre tensión y deformación, (e ) la carga sobre el sistema se aplica en la capa superior sobre superficies circulares con presión uniforme sobre dicha área y (f), la cama de arena no produce aporte estructural, por lo que no se incluyó en la estructura del pavimento. La Tabla 5 resume las propiedades de los materiales empleados en el análisis mecanicista. La solicitación de tránsito se caracterizó mediante el eje estándar: eje simple de rueda doble, carga de rueda de $20 \mathrm{kN}$, presión de inflado de $520 \mathrm{kPa}$ y una distancia entre ejes de rueda doble de $30 \mathrm{~cm}$. Las tensiones, deformaciones y deflexiones se evaluaron en la subrasante en el punto medio bajo las ruedas del eje estándar.

\begin{tabular}{|c|c|c|c|c|}
\hline \multirow[b]{2}{*}{ Capa } & \multicolumn{2}{|c|}{ Módulo de elasticidad } & \multicolumn{2}{|c|}{ Razón de Poisson } \\
\hline & $\begin{array}{l}\text { Valor } \\
(\mathrm{MPa})\end{array}$ & Referencia & Valor & Referencia \\
\hline Adoquines & 1500 & Shackel (2000) & 0,30 & $\begin{array}{l}\text { Judicky et al. } \\
\text { (1996) }\end{array}$ \\
\hline $\begin{array}{l}\text { Cama de } \\
\text { arena }\end{array}$ & $\begin{array}{l}\text { Sin aporte } \\
\text { estructural }\end{array}$ & Shackel (1980) & $\begin{array}{l}\text { Sin aporte } \\
\text { estructural }\end{array}$ & Shackel (1980) \\
\hline $\begin{array}{l}\text { Base } \\
\text { granular }\end{array}$ & $\begin{array}{l}\text { Ecuación } \\
\text { Shell }\end{array}$ & Reyes (2004) & 0,35 & $\begin{array}{l}\text { Judicky et al. } \\
\text { (1996) }\end{array}$ \\
\hline $\begin{array}{l}\text { Sub- } \\
\text { rasante }\end{array}$ & $\begin{array}{l}\text { Módulo } \\
\text { Resiliente }\end{array}$ & $\begin{array}{l}\text { Ryntathiang et } \\
\text { al. (2006) }\end{array}$ & 0,35 & $\begin{array}{l}\text { Ryntathiang et } \\
\text { al. (2006) }\end{array}$ \\
\hline
\end{tabular}

\section{Tensión de compresión en la sub-rasante}

En las Figuras $4 a, 4 b$ y $4 c$ se muestran las tensiones de compresión calculadas de acuerdo a la metodología antes descrita. Se incluye la tensión admisible de compresión de la subrasante ( $\sigma_{\text {adm }}$ en MPa) propuesta por Armijos (2011). Estas tensiones admisibles varían entre 0,07 MPa para CBR del $4 \%$ y 0,21 MPa para un CBR del 20\%. Los resultados muestran que de manera sistemática los diseños del método británico presentan los menores niveles de compresión de la subrasante y se encuentran siempre bajo los resultados obtenidos por el resto de los métodos y además bajo los valores admisibles. Para todos los niveles de tránsito estudiados este método mantiene las tensiones de compresión prácticamente constantes para cualquier valor de CBR y con un valor máximo de 0,05 MPa para tránsito "bajo", "medio" y 0,01 MPa para tránsito "alto".

En el nivel de tránsito "bajo" (Figura 4a) se aprecia que para CBR inferiores al 9\%, con excepción del método británico, los demás métodos sobrepasan los niveles admisibles de compresión de la subrasante. Ocurre lo contrario para CBR mayores al $9 \%$, donde las tensiones obtenidas se encuentran por debajo de la tensión admisible. Además se observa que a partir de un CBR del $10 \%$, las tensiones obtenidas convergen a $0,14 \mathrm{MPa}$, exceptuando el modelo del Reino Unido, en el cual la tensión máxima obtenida alcanzó los 0,06 MPa.

Para niveles de tránsito "medio" (Figura 4b), las tensiones obtenidas en los diseños realizados según los métodos del Reino Unido, Japón y Estados Unidos, se encuentran por debajo de las tensiones admisibles en todo el rango de valores de CBR. Esto no ocurre con los métodos de Australia e India, los cuales presentan resultados mixtos en que: para CBR menores al 9\% las tensiones están sobre los valores admisibles y para CBR mayores a $9 \%$ los resultados se encuentran por debajo de la tensión admisible. Para niveles de tránsito "alto" (Figura 4c) la compresión en la subrasante obtenida para todos los métodos utilizados y para todo el rango de CBR, es inferior a los niveles admisibles. 
Figura 3. Espesor de base granular para nivel de tránsito (a) bajo, (b) medio y (c) alto (Fuente: Elaboración Propia, 2012).
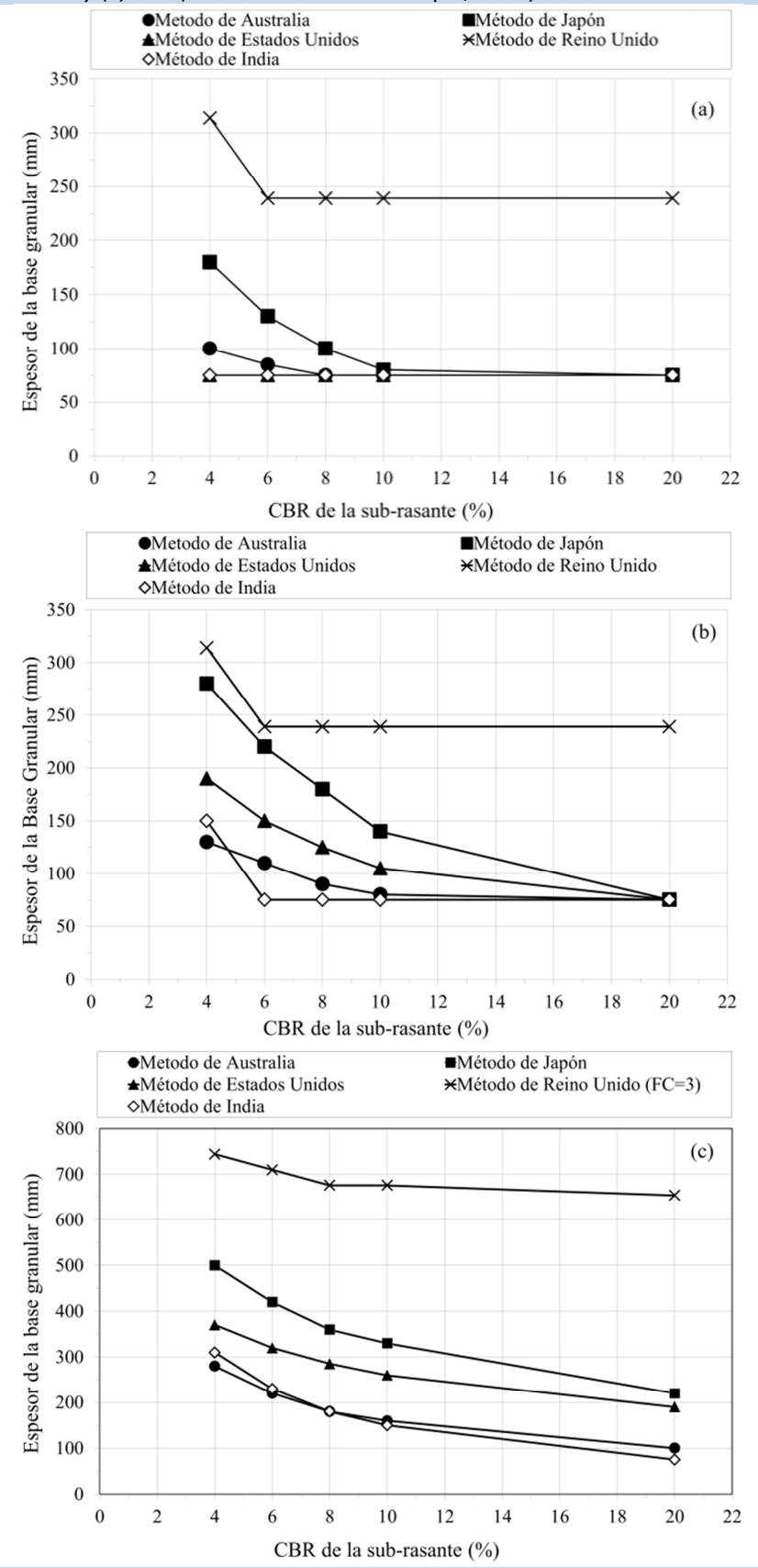

\section{Deformación vertical de la subrasante}

En las Figuras $5 a, 5 b$ y $5 c$ se grafican las deformaciones elásticas en la subrasante calculadas con el software BISAR V3.0. Además se incluye la deformación admisible de la sub-rasante $\left(\varepsilon_{\text {adm }}\right)$ propuesta por Shell (Reyes, 2004). Para los valores característicos de cada nivel de tránsito analizado, la ecuación 5 arroja deformaciones admisibles entre 0,6 y $1,9 \times 10^{-3} \mathrm{~mm} / \mathrm{mm}$. Los resultados muestran que para los tres niveles de tránsito analizados, los pavimentos diseñados con el método británico presentan deformaciones inferiores a los obtenidos usando los demás métodos de diseño, e inferiores a los valores admisibles calculados con la ecuación 5 : $1,9 \times 10^{-3}, 1,1 \times 10^{-3}$ y $0,6 \times 10^{-3}$ $\mathrm{mm} / \mathrm{mm}$ para niveles de tránsito "bajo" (Figura $5 \mathrm{a}$ ), "medio" (Figura 5b) y "alto" (Figura 5c) respectivamente.
Figura 4. Compresión en la sub-rasante para tránsito (a) bajo, (b) medio y (c) alto (Fuente: Elaboración Propia, 2012).

\begin{tabular}{lll} 
& $\begin{array}{l}\text {-Método de Australia } \\
\text {-Método de Estados Unidos } \\
\text {-Método de India }\end{array}$ & $\begin{array}{l}\text { \#Método de Japón } \\
\text { *Método de Reino Unido } \\
\text { +øadm (Armijos, 2011) }\end{array}$ \\
\hline 0,25 & & \\
\hline
\end{tabular}
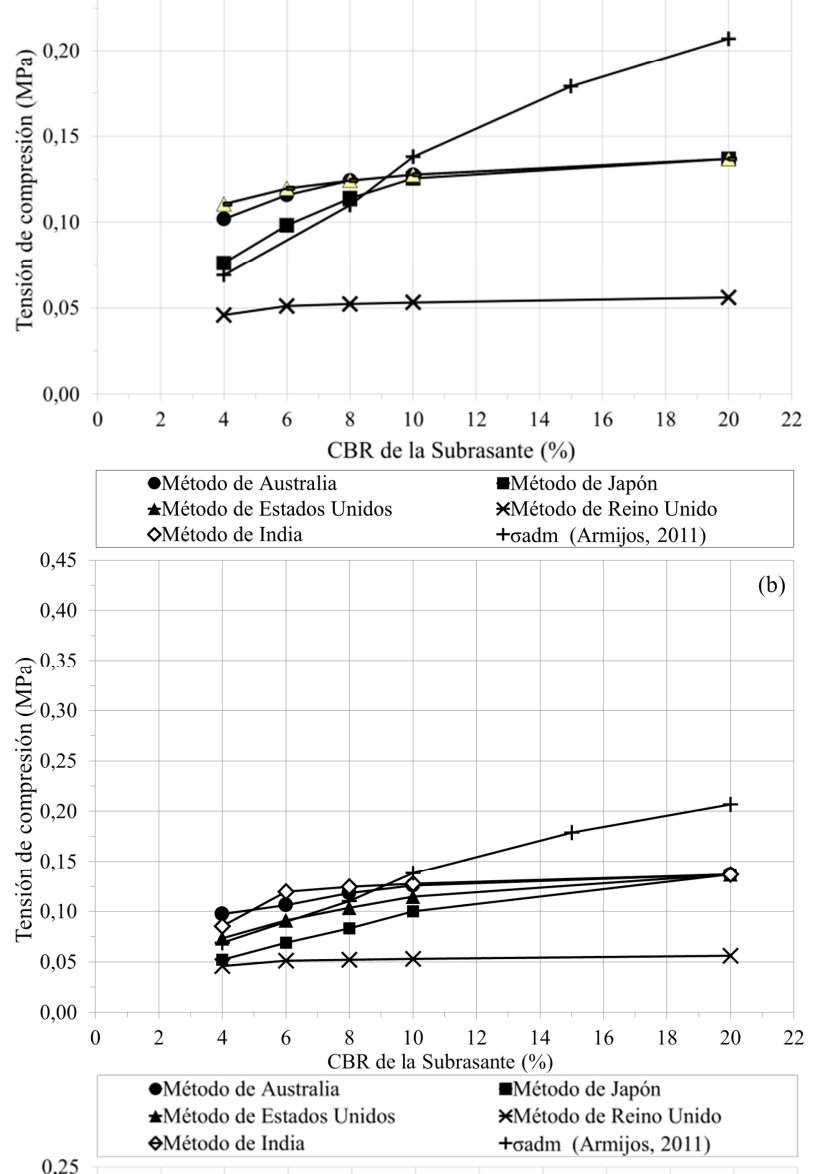

0,25

(c)

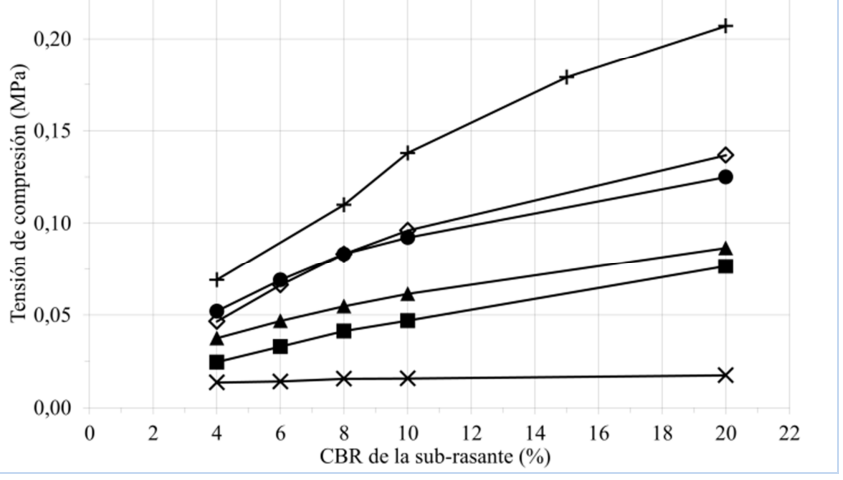

En el método de Japón se observa que tanto para el nivel de tránsito "medio" como para el "alto", las deformaciones presentan un comportamiento estable y cercano al valor admisible para todo el rango de CBR usado en el diseño. Para el

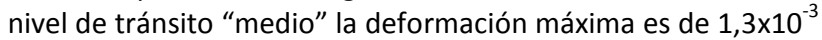
$\mathrm{mm} / \mathrm{mm}$ para CBR $4 \%$ y la mínima es de $1 \times 10^{-3} \mathrm{~mm} / \mathrm{mm}$ para CBR 20\%. Para el tránsito "alto", la deformación es prácticamente constante en torno al $0,6 \times 10^{-3} \mathrm{~mm} / \mathrm{mm}$. Para el nivel de tránsito "medio" los resultados para CBR inferiores al $19 \%$ en todos los métodos con excepción del británico, las deformaciones obtenidas se encuentran por sobre el nivel admisible. Solo para CBR del $20 \%$, las deformaciones se mantienen bajo el nivel admisible en todos los métodos. Similar 
es el comportamiento en el nivel de tránsito "alto", en el cual la deformación admisible es sobrepasada por todos los diseños excepto por aquellos elaborados con el método británico.

Figura 5. Deformación en la sub-rasante para tránsito (a) bajo, (b) medio y (c) alto (Fuente: Elaboración Propia, 2012).

\begin{tabular}{|c|c|c|}
\hline & $\begin{array}{l}\bullet \text { Método de Australia } \\
\text { \# Método de Estados Unidos } \\
\text { ○Método de India }\end{array}$ & $\begin{array}{l}\text { M Método de Japón } \\
\text { *Método de Reino Unido } \\
\text { +عz adm Shell (1977) }\end{array}$ \\
\hline$\left[\begin{array}{l}2,40 \\
2,20\end{array}\right.$ & & (b) \\
\hline
\end{tabular}

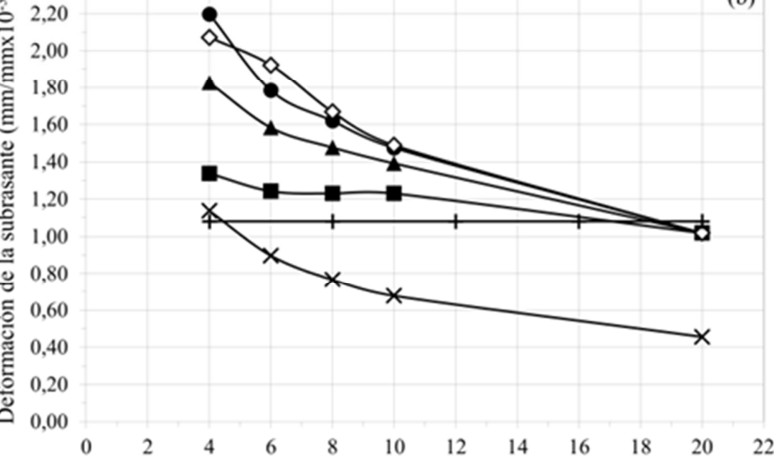

CBR de la Subrasante (\%)
$\bullet$ @étodo de Australia
घMétodo de Japón

$\overbrace{}^{3} 2,60$

$\times$

臧 2,200

咅 2,00

ญ 1,60

藏 1,40

今े 1,20

ㅍ 1,00

을 0,80

은 0,60

冚 0,40

0,20

0,00

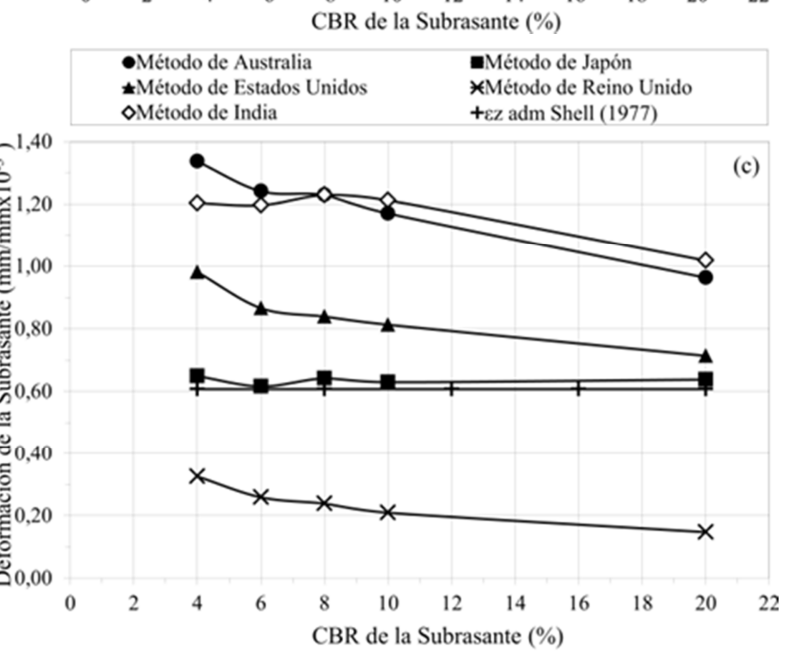

$\varepsilon_{\text {adm }}=0,028 \mathrm{~N}_{80}^{0,25}$

$\Delta_{\mathrm{adm}}=26,32202 \mathrm{~N}_{80}^{-0,2438}$

\section{Deflexión de la subrasante}

En las Figuras 6a, 6b y 6c se muestra la deflexión elástica en la sub-rasante calculada para cada método de diseño y la deflexión admisible $\left(\Delta_{\mathrm{adm}}\right.$, en $\left.\mathrm{mm} / \mathrm{mm} \times 10^{-3}\right)$, calculada con la Ec. 6.

Figura 6. Deflexión de la sub-rasante para tránsito (a) bajo, (b) medio y (c) alto (Fuente: Elaboración Propia, 2012).

\begin{tabular}{|c|c|}
\hline -Método de Australia & -Método de Japón \\
\hline $\begin{array}{l}\text { Método de Estados Unidos } \\
\text {-Método de India }\end{array}$ & $\begin{array}{l}\text { *Método de Reino Unido } \\
+\Delta \text { adm Huang }\end{array}$ \\
\hline
\end{tabular}

\begin{tabular}{|l|l|l|l|}
\hline $2,50 \mid$ & (a) \\
\hline
\end{tabular}

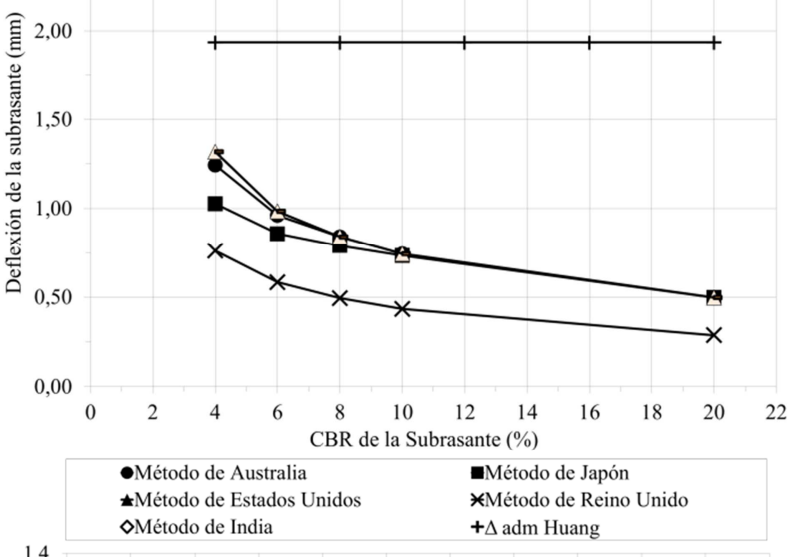

(b)

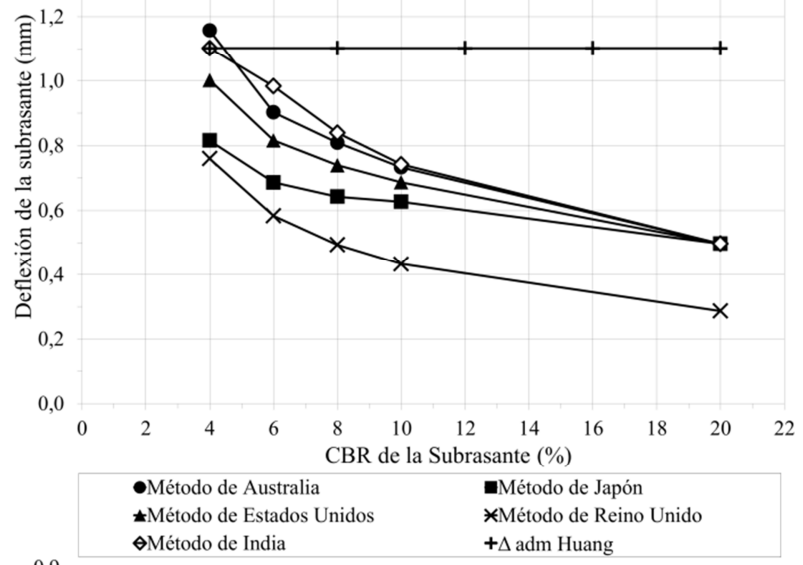

0,9

$\diamond$ Método de India $\quad+\Delta$ adm Huang

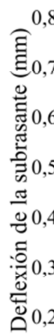

Para nivel de tránsito "bajo" (Figura 6a), los diseños obtenidos por todos los modelos, se encuentran por debajo del nivel admisible. Se alcanza un máximo entre $0,7 \mathrm{~mm}$ y $1,3 \mathrm{~mm}$ para un CBR del $4 \%$ y un mínimo entre 0,3 y $0,5 \mathrm{~mm}$ para un CBR del 
20\%. Para nivel de tránsito "medio" (Figura 6b), los diseños obtenidos por todos los modelos, se encuentran por debajo del límite admisible con excepción del método australiano en el cual para un CBR del $4 \%$ alcanza una deflexión de 1,2 mm. Para el nivel de tránsito "alto" (Figura 6c) los métodos de Australia e India exceden la deflexión admisible cuando los CBR de la subrasante son inferiores al $8 \%$. El resto de métodos para CBR sobre el $4 \%$ exhiben deformaciones inferiores a la admisible y un valor mínimo entre 0,15 y 0,5 mm para CBR del $20 \%$. En general, los resultados muestran que los diseños obtenidos con el método británico presentan las menores deflexiones en todos los niveles de tránsito $y$ en todo el rango de CBR considerado en los diseños. Además son inferiores a los niveles admisibles.

\section{Conclusiones}

Este trabajo tuvo el propósito de analizar los métodos de diseño de pavimentos de adoquines de hormigón propuestos en Australia, Japón, Estados Unidos, Reino Unido e India. Se obtuvieron los espesores de base granular con cada uno de los métodos citados para 25 escenarios de diseño y se evaluó el desempeño de cada pavimento usando un enfoque mecanicista, en cual se analizaron las tensiones, deformaciones y deflexiones. Se concluyó que el método británico permite obtener diseños con espesores mayores al resto de los métodos analizados y dentro del rango de CBR entre $4 \%$ y $10 \%$, pero con un desempeño sustancialmente superior al que se logra con el resto de métodos de diseño. En segundo lugar se encuentra el método Japonés el cual cumple con la mayoría de los criterios de desempeño para todos los niveles de tránsito y CBR analizados.

Cuando la subrasante existente posee un CBR $>10 \%$, todos los métodos de diseño arrojan espesores iguales al mínimo y con un buen desempeño. Es decir, tensiones, deformaciones $y$ deflexiones se encuentran bajo los valores admisibles. Por tanto, proyectar un pavimento con cualquier método de diseño y subrasantes de buena calidad, lleva a diseño mínimo de buen desempeño, independientemente del método. La mayor variabilidad en espesores y desempeño, entre los métodos analizados ocurre cuando el CBR de la subrasante es inferior al $10 \%$. En esta caso, se puede llegar a diferencias de hasta 200 $\mathrm{mm}$ para los CBR más bajos y para el nivel de tránsito más bajo. Esta variabilidad en espesores aumenta conforme aumenta el nivel de tránsito, pudiendo llegar hasta los $700 \mathrm{~mm}$.

La elección de uno u otro método de diseño lleva a grandes diferencias en espesores, pero el impacto final sobre el pavimento se verá reflejado esencialmente en el desempeño. En este sentido, si bien el método británico proporciona mayores espesores, posee un desempeño sustancialmente superior que resto de métodos en términos de tensiones, deformaciones y deflexiones en la subrasante. El método británico posee ventajas respecto de los otros métodos analizados para solicitaciones de tránsito elevadas. Por el contario, para solicitaciones de tránsito bajas puede sobreestimar espesores, pero asegurando en todo caso un buen desempeño.

En este trabajo no se incluyó un análisis del factor de equivalencia de bases utilizado por el método de diseño del Reino Unido, el cual determina que el espesor de una base granular es 3 veces superior a una base enriquecida con cemento. Puesto que la magnitud de este factor depende de la dosificación de cemento y de su resistencia, es necesario analizar la magnitud de dicho factor para bases tratadas con cemento nacionales equivalentes en dosificación, determinar su resistencia y el efecto que tendría en el diseño de espesores y en el desempeño del pavimento siguiendo el procedimiento de análisis empleado en este trabajo. Asimismo, no se consideró el efecto de rigidización del conjunto adoquín / cama de arena, el cual tiende a reducir las tensiones en la base. Por lo tanto, para realizar un análisis más detallado de los métodos de diseño se recomienda verificar las mismas estructuraciones aquí propuestas pero considerando diversos espesores de adoquines y cama de arena, y verificarlos mediante ensayos estáticos y dinámicos de carga.

\section{Referencias}

AASHTO (1993). American Association of State Highway and Transportation Officials. AASHTO Guide for Design of Pavement Structures 1993. American Association of State Highway and Transportation Officials: Washington, DC.

Armijos, V. (2011). Estudio del diseño estructural y constructivo de pavimentos articulados en base a bloques de asfalto", Tesis de Magíster en Ciencias de la Ingeniería, Pontificia Universidad Católica de Chile [Tesis en Español].

ASCE 58-10. (2010). American Association of Civil Engineers. Structural design of interlocking concrete pavement for municipal streets and roadways. American Association of Civil Engineers: Reston, Virginia, Estados Unidos.

Barthou, J. (1991). Pavimentos de Adoquines. Manual de diseño y construcción. Santiago: Instituto Chileno del Cemento y el Hormigón.

BSI 7533-1:2001 (2001a). British Standard Institution. Guide for the structural design of heavy duty pavements constructed of clay pavers or precast concrete paving blocks. British Standard Institution: London, UK.

BSI 7533-2:2001 (2001b). British Standard Institution. Guide for the structural design of lightly trafficked pavements constructed of clay pavers or precast concrete paving blocks. British Standard Institution: London, UK.

Clifford, J.M (1987). Structural Design of Segmental Block Pavements for Southern Africa. South Africa: National Institute for Transport and Road Research.

Eisenmann, J. \& Leykauf, G. (1988). Design of Concrete Block Pavements in FRG. 4th International Conference on Concrete Block Paving, Roma. 
Houben, L., Van der Vlist A., Dekkers, J., Duivenvoorden, J., Van der Kooster, J., Leewis, M. \& Nonneman, D. (1986). The Dutch Design Method for Concrete Blocks Road Pavement. 3rd International Conference on Concrete Block Paving, Melbourne.

Judycki, J., Alenowicz, J. \& Cyske, W. (1996). Structural Design of Concrete Block Pavement Structures for Polish Conditions. 7th International Conference on Concrete Block Paving, Tel Aviv.

Knapton, J. (2008). The Structural Design of Heavy Duty Pavements for Ports and Others Industries. Leicester: British Precast Concrete Federation Ltd.

Livneh, M., Ishai, I. \& Nesichi, S. (1988). Development of a Pavement Design Methodology for Concrete Block Pavements in Israel. 4th International Conference on Concrete Block Paving, Roma.

MINVU (2008). Código de normas y especificaciones técnicas de obras de pavimentación. Santiago: Ministerio de Vivienda y Urbanismo.

Miura, Y., Takaura, M. \& Tsuda, T. (1984). Structural design of concrete blocks pavements by CBR method and its evaluation. 2nd International Conference on Concrete Block Paving, Delf.

Morrish, C. (1980). Interlocking concrete paving - the state of the art in Australia. 1st International Conference on Concrete Block Paving, Newcastle.

MOP (2012). Instrucciones y Criterios de Diseño, Manual de Carreteras, Vol. 3. Santiago: Ministerio de Obras Públicas.

Panda, B. \& Ghosh, A. (2002). Structural Behavior of Concrete Blocks Paving. I: Sand in Bed and Joints. Journal of Transportation Engineering, 128(2), 123-129.

Rada, G., Smith, D., Miller, J. \& Witczak, M. (1990). Structural Design of Concrete Block Pavements. Journal of Transportation Engineering, 116(5), 615-635.

Reyes, F. (2004). Diseño Racional de Pavimentos. Bogotá: Editorial Escuela Colombiana de Ingeniería.

Read, J. \& Whiteoak, D. (2003). The Shell Bitumen Handbook. London: Thomas Telford.

Rollings, R. (1984). Corps of engineers design method for concrete block pavements. 2nd International Conference on Concrete Block Paving, Delf.

Ryntathiang, T., Mazumdar, M. \& Pandey, B. (2006). Concrete Block Pavement for Low Volume Roads. 8th International Conference on Concrete Block Paving, San Francisco.

Shackel, B. (1980). The Performance of Interlocking Block Pavements under Accelerated Trafficking. 1st International Conference on Concrete Block Paving, Newcastle.

Shackel, B., O'Keeffe, W. \& O'Keeffe, L. (1993). Concrete Block Paving Tested as Articulated Slabs. 5th International Conference on Concrete Pavement Design and Rehabilitation, Indiana.

Shackel, B. (2000). The Development and Application of Mechanistic Design Procedures for Concrete Block Paving. 6th International Conference on Concrete Block Paving, Tokyo.
Shackel, B. \& Lim, D. (2003). Mechanism of Paver Interlock. 7th International Conference on Concrete Block Paving, Tel Aviv.

Silfwerbrand, J. \& Wäppling, M. (2000). Parameter on the Design of Concrete Block Pavements. 9th International Conference on Concrete Block Paving, Tokyo. 